\title{
Chemorheology of Cross-linked Polymers. Chemorheological Treatment of Cross-linked Polymers
}

\author{
Kenkichi Murakami and Saburo Tamura \\ Chemical Research Institute of Non-aqueous Solutions, \\ Tohoku University, Sendai, Japan.
}

(Received September 24, 1970)

\begin{abstract}
Stress relaxation mechanisms were investigated, on three types of EPT polymers, in air at $109^{\circ} \mathrm{C}$. These polymers differ only in the structure of the crosslinkage in which there is a carbon-carbon bond, a polysulfide linkage (-Sx-) or a monosulfide linkage (-S-).

All the stress relaxation of a peroxide-cured EPT polymer is not due to the oxygeninduced cleavage of the main chain but to a physical flow. In the case of a sulfur-cured EPT polymer, the relaxation curve is divided into three straight lines by using the procedure $X$ when $\log f(t) / f(0)$ is plotted against $t$. It was concluded that this graph could be attributed to an interchange reaction between the polysulfide linkage and an oxidative cleavage of the monosulfide linkage. On the other hand, a TT-cured EPT polymer gave a plot with a straight line. This stress relaxation could be explained by an oxidative cleavage of the monsulfide linkage.

Further, we have investigated the degree of agreement between the theoretical relation proposed by us and experimental results relating to TT-cured natural rubber. In this cross-linked polymer, both scissions of main chain and cross-linkage occur at the same time.

KEY WORDS Chemical Stress Relaxation/Peroxide-Cured/Sulfur-

Cured / TT-Cured / EPT / Interchange Reaction / Cross-linking

Density / Procedure $X$ / Natural Rubber /
\end{abstract}

In the degradation of cross-linked polymers, it is an urgent problem to determine whether the scission of the cross-linked polymer occurs along the main chains or at the cross-linkages.

While both extreme cases have been studied theoretically by A. V. Tobolsky, et al. ${ }^{1,2}$ the cases that both scissions occur at the same time remain unsolved owing to the difficulty of their analysis.

This paper deals with the theoretical and experimental results of the chemorheological treatment of cross-linked polymers concerning their scission mechanisms.

\section{EXPERIMENTAL}

Ethylene-propylene terpolymer (EPT, Royalene 301, Sumitomo Chemical Industry Ltd.) was cold milled with the curing ingredients as described in Table I. Table I also shows curing conditions. Thin sheets (about $0.5 \mathrm{~mm}$ ) of the milled polymer were pressed under $200 \mathrm{~kg} / \mathrm{cm}^{2}$ at the curing temperature.

Three kinds of EPT polymer (A, B, and C) differing only in the structure of the cross-linkage were prepared. It is evident that the crosslinking site, in these samples, consists of a car-

Table I. Cross-linking agents and preparing of cured EPT polymer ${ }^{a}$

\begin{tabular}{lrrc}
\hline \multicolumn{1}{c}{ Sample no. } & A & B & C \\
\hline Rubber & 100 & 100 & 100 \\
Sulfur & & 2 & \\
Zinc oxide & & 5 & 5 \\
Stearic acid & & 2 & 1 \\
TT $^{\mathrm{b}}$ & & 1 & 3.5 \\
MBT $^{\mathrm{c}}$ & 2 & 0.5 & \\
DCP $^{\mathrm{d}}$ & & & \\
\hline Curing time, min & 30 & 20 & 40 \\
\hline
\end{tabular}

a Curing temp, $150^{\circ} \mathrm{C}$.

b Tetramethylthiuramdisulfide.

c Mercaptobenzothiazole.

d Dicumyl peroxide. 
bon-carbon bond, a polysulfide linkage $\left(-S_{x^{-}}\right)$ and a monosulfide linkage $\left(-\mathrm{S}_{-}\right)$for $\mathrm{A}, \mathrm{B}$, and $\mathrm{C}$, respectively. All the samples were extracted with acetone during $72 \mathrm{hr}$ and dried in vacuo.

The stress relaxation apparatus used here was made in our laboratory, and its mechanism is a usual strain-gauge system.

The network chain density $n(0)$ was estimated by the following equation.

$$
f(0)=n(0) R T\left(a-a^{-2}\right)+C\left(1-a^{3}\right)
$$

In this expression $f(0)$ is the initial stress, $R$ is the gass constant, $T$ is the absolute temperature, $a$ is the extention ratio, and $C\left(1-a^{3}\right)$ corresponds to the stress based on the physical network chains.

\section{RESULTS}

The stress relaxation of dicumyl peroxidecured EPT (sample A) was measured at $109^{\circ} \mathrm{C}$ in air, in nitrogen, and in air containing a proper amount of antioxidant. These stress re- laxation curves (relative stress $f(t) / f(0)$ vs. time $\log t)$ are shown in Figure 1. From this figure, it is seen that the descending curves indicate almost the same inclination in the three samples. When we consider both the chemical structure of sample $\mathrm{A}$ and the experimental conditions, it seems to be very reasonable to assume that the relaxation mechanism of the sample is clearly due to a physical relaxation, that is, a physical flow of the polymer chains.

Also, the stress relaxation of sample A having the initial density $n(0)=0.53 \times 10^{-4} \mathrm{~mol} / \mathrm{m} l$ and $1.45 \times 10^{-4} \mathrm{~mol} / \mathrm{m} l$ was measured in the air at $109^{\circ} \mathrm{C}$. And those of samples $\mathrm{B}$ and $\mathrm{C}$ having $n(0)+1.39 \times 10^{-4} \mathrm{~mol} / \mathrm{ml}$ and $0.62 \times 10^{-4} \mathrm{~mol} / \mathrm{ml}$ respectively were measured under the same conditions. The relation of relative stress vs. time is shown in Figure 2. Because Figures 1 and 2 have been established under the same conditions, the superposed portions of the curves in samples $B$ and $C$ with that of sample $A$ are considered to be due to a physical relaxation. From Figure 2 , it was found that the stress decay due to a

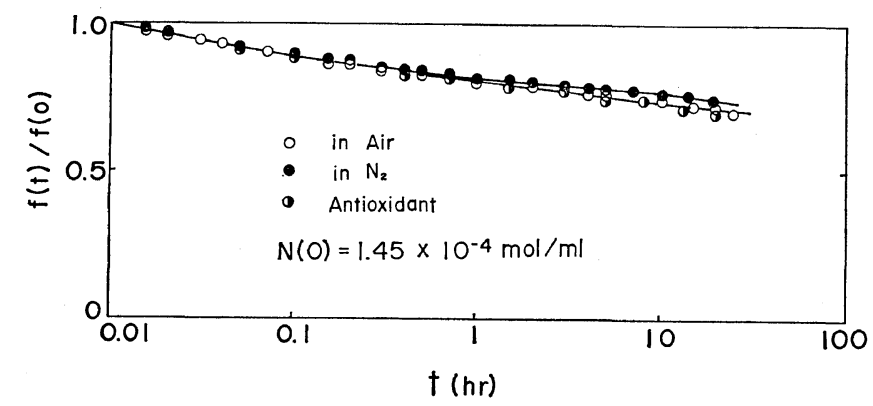

Figure 1. Stress relaxation of dicumyl peroxide-cured EPT (sample no. A) in the air, under nitrogen and in the air containing a proper amount of antioxidant at $109^{\circ} \mathrm{C}$.

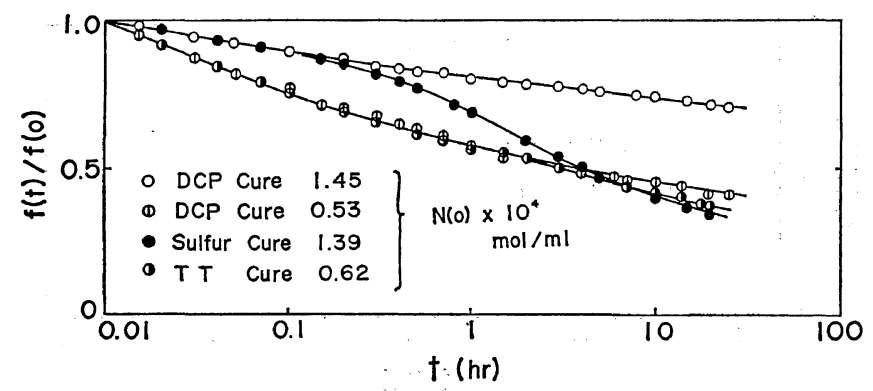

Figure 2. Stress relaxation of samples no. A, no. B (sulfur-cured ETP), and no. C (TT-cured EPT) in the air at $109^{\circ} \mathrm{C}$. 


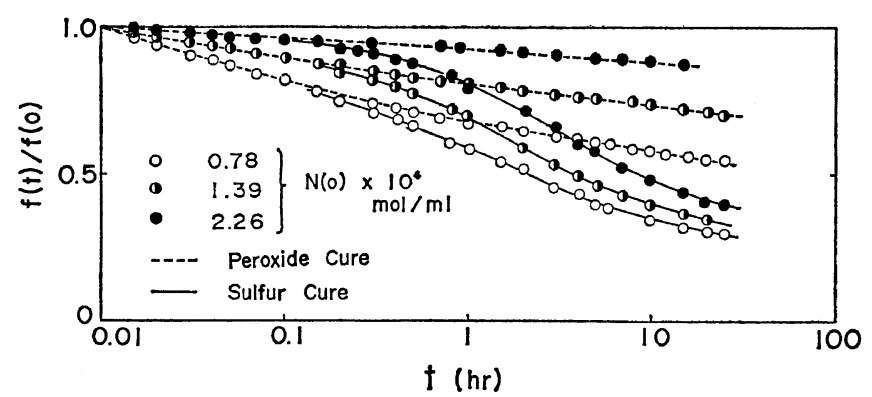

Figure 3. Stress relaxation of three groups of samples no. A and no. B having the same value of $n(0)$ in the air at $109^{\circ} \mathrm{C}$.

physical relaxation at the initial stages is independent of the kind of cross-linkages if the network-chain density of these polymers is equivalent. For further identification, we have carried out the following experiments. Materials in which the kinds of cross-linkages are different but with identical initial densities of $n(0)$ were prepared. In these materials, the cross-linkages $\mathrm{C}-\mathrm{C}$ bond and polysulfide linkage $\left(-\mathrm{S}_{x^{-}}\right)$, and $n(0)$ of the samples having the same density $0.78 \times 10^{-4} \mathrm{~mol} / \mathrm{ml}, 1.39 \times 10^{-4} \mathrm{~mol} / \mathrm{ml}$, and $2.26 \times$ $10^{-4} \mathrm{~mol} / \mathrm{m} l$ respectively. The stress relaxation curves obtained from these samples are shown in Figure 3. From Figure 3, it is clear that as noted above, with a nearly equal value of $n(0)$ in these samples, those physical relaxations at the initial stage are the same and they overlap independently of the kinds of cross-linkages.

When we look at the Figure 2 once again for the sulfur-cured EPT (sample B), physical relaxation is noticed at the initial stage which is then followed markedly by chemical relaxation. As the relaxation curve of TT-cured EPT (sample C) has been superposed with that of sample A almost all over the ranges of the observation time, the case of sample $\mathrm{C}$ seems almost based on physical relaxation. As for the structures of the cross-linking sites of sample $\mathrm{C}$, since they contain mono- and di-sulfide, this sample is regarded as stable.

In order to discuss the mechanisms of chemical relaxation in detail, portions of the stress decay based upon the physical flow were reduced from the original stress relaxation curves of samples $\mathbf{B}$ and $\mathbf{C}$ in Figure 2, and their pure chemical stress relaxation curves were replotted as in Figure 4 . Since it is confirmed by reference $^{3}$ that the main chains of EPT are very stable to oxidative scission under the experimental conditions used here, it can be safely assumed from Figure 4 that a few cleavages would have to occur at the cross-linkage in $\mathrm{C}$ and the interchange reactions mostly at the cross-linking sites in $\mathbf{B}$.

Then when two chemical stress relaxation curves in Figure 4 were replotted as $\log f(t) / f(0)$ $v s$. time, these resulted in Figure 5. That is, from such plotting, a fines traight line was ob-

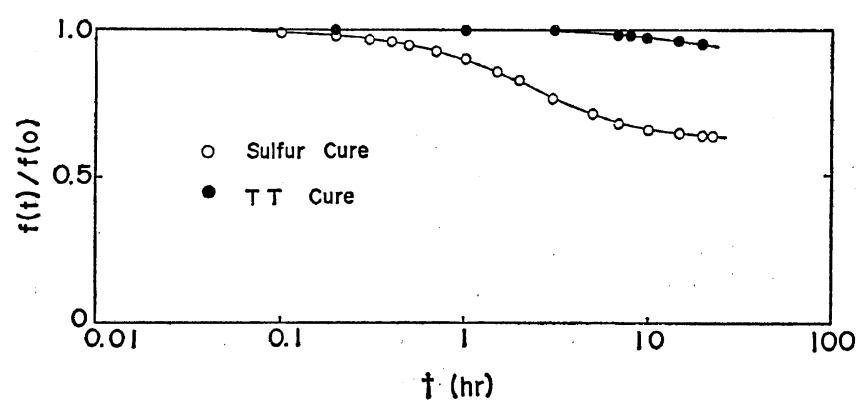

Figure 4. Pure chemical stress relaxation of samples no. $B$ and no. $C$ obtained from Figure 2. 
tained for sample $\mathrm{C}$, on the contrary, because there remained identified portions of curves for sample $B$ in the short time region as shown in the figure. We used our suggested Procedure $X^{4}$ and repeated it until we obtained a straight line in the whole time scale region. On the other hand, the portions of the stress decay based on the physical flow of polymer chains were reduced from the original stress relaxation curves of sulfur-cured EPT in Figure 3. The results are shown in Figure 6 as the plot of $\log f(t) / f(0)$ vs. $t$.

A. V. Tobolsky, et al. ${ }^{3}$ have recently carried out chemical relaxation in respect of sample B, under approximately similar experimental conditions as ours, and the experimental results they obtained are shown in Figure 7. This figure indicates that the stress decay based on the interchange mechanism of a polysulfide cross-linkage occurs rapidly at the initial stage of the relaxation time, but after some hours

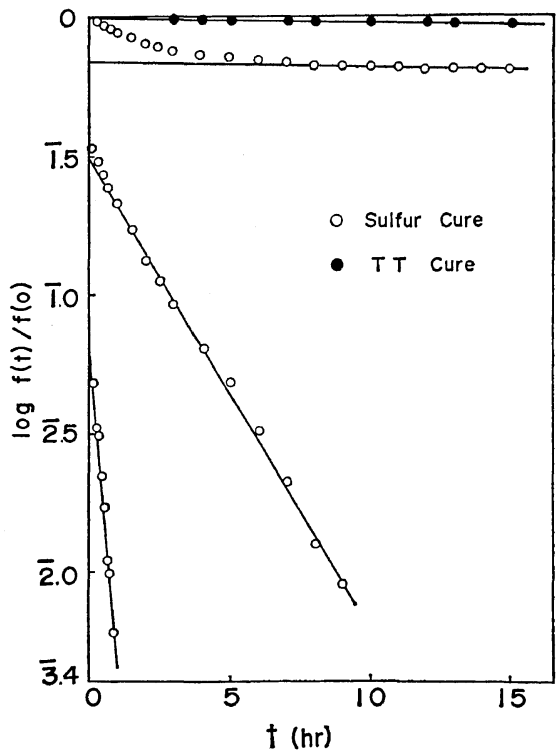

Figure 5. Replot as $\log f(t) / f(0) v s$. time of two chemical stress relaxation curves in Figure 4.

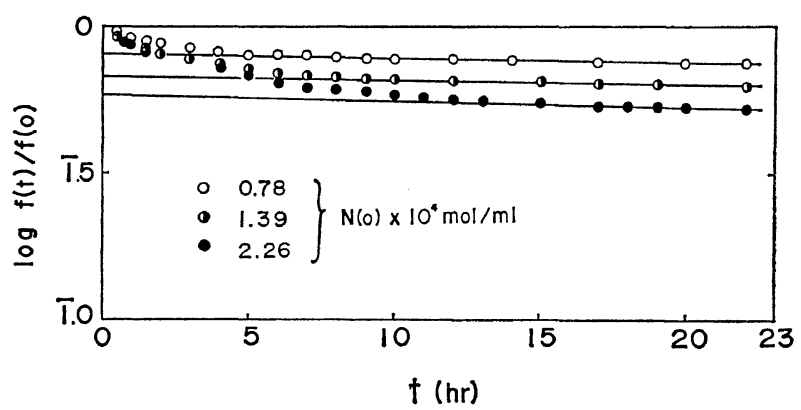

Figure 6. Replot as $\log f(t) / f(0)$ vs. time of three chemical stress relaxation curves in Figure 3.

the stress decay curve becomes parallel to the abscissa owing to thermally stable mono- and di-sulfide cross-linkages. These results differ from ours in Figures 5 and 6 and these differences merit comments.

(I) In our experimental results, that is, in the case of sample B (sulfur-cured EPT polymer) in Figures 5 and 6, the straight lines on the long time side are not parallel to the abscissa but have slightly descendent similar slopes. Which results are correct?

(II) The stress relaxation curve relating to sample $B$ is divided into three fine straight lines by using the Procedure $X$ as shown in Figure 5 . Can this be explained?

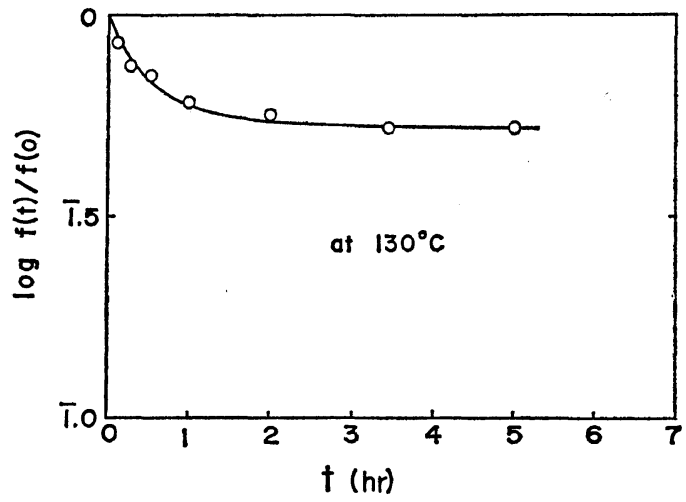

Figure 7. Tobolsky, et al.'s, data of stress relaxation of sample no. $B$ in the air at $130^{\circ} \mathrm{C}$. 


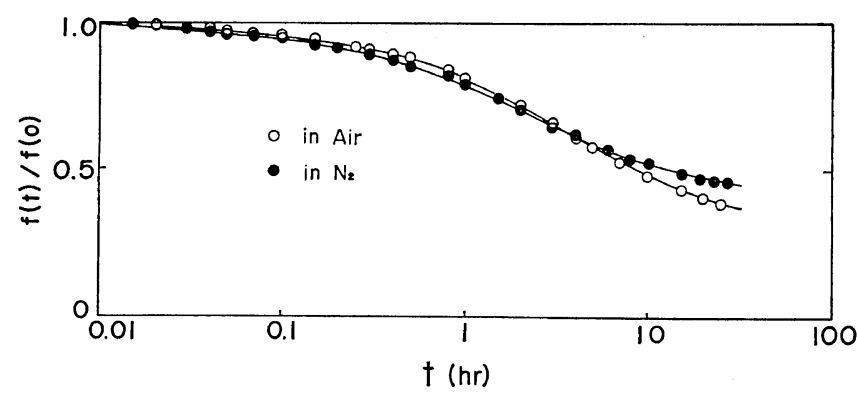

Figure 8. Stress relaxation curves of sample no. $\mathbf{B}$ in the air and under nitrogen at $109^{\circ} \mathrm{C}$.

(III) In Figure 5, both slopes of a straight line for sample $\mathrm{C}$ and the uppermost straight line of the three for sample $B$ are identical. Further in Figure 6, all the slopes of the three straight lines for sample $B$ are independent of the cross-linking density, $n(0)$. Why?

The chemical stress relaxation curves of sample $\mathrm{B}$ at $109^{\circ} \mathrm{C}$ in both air and nitrogen are shown in Figure 8. If these stress decays were based only on the interchange of polysulfide cross-linkage, then they must be independent of the surroundings. But on the long time side, they are somewhat more descendent in the air than in the nitrogen in Figure 8 . We may consider that in sample $\mathrm{B}$, in addition to the interchange reaction of the polysulfide linkages, an oxygen-induced cleavage of the mono- and di-sulfide linkages in the air could explain this behaviour.

\section{DISCUSSION}

We will explain the above results by using mathematical expressions in more detail. Supposing a scission occurs only at the cross-linkage in the polymer network, we replace the number of initial cross-linkages by $C(0)$, the number of scissions after $t$ hours by $q(t)$ and the proportional constant by $k$. The following equation is then assumed.

$$
\frac{\mathrm{d} q(t)}{\mathrm{d} t}=k\{C(0)-q(t)\}
$$

solving eq 1 , eq 2 is obtained.

$$
q(t)=C(0)\left\{1-\mathrm{e}^{-k t}\right\}
$$

If $f(t) / f(0)$ is the relative stress and $n(0)$ the initial network-chain density when the scission occurs only at the cross-linkages, then the following equation is valid:

$$
\frac{f(t)}{f(0)}=1-\frac{2 q(t)}{n(0)}
$$

In the ideal network of the chains the eq 4 is obtained.

$$
2 C(0)=n(0)
$$

Therefore eq 5 is deduced from the above equations.

$$
\frac{f(t)}{f(0)}=\mathrm{e}^{-l t}
$$

When a scission occurs only at the cross-linkage in the stress-relaxation curve, it is expressed by a simple Maxwellian decay.

From previous studies, ${ }^{9}$ it has been known that the stress relaxation mechanism of crosslinked polymer in which only interchange reaction occurs is represented by one Maxwellian decay curve. Recently, however, from additional treatment in which the strength of interchangeable bonds is heterogeneous instead of being homogeneous in the previous assumption, more exact theoretical results have been obtained as follows.

Suppose a fraction $\boldsymbol{P}_{w}$ of these bonds were weak linkages. We can classify the network chains as $N_{0}, N_{1}, N_{2}, \ldots, N_{\bar{x}}$ chains per cubic centimeter containing $0,1,2, \ldots, \bar{x}$ weak linkages. Since the total network chains per cubic centimeter of all kinds is $n(0)$, each network 
chain is represented by the following equations.

$$
\begin{aligned}
& N_{0}=n(0)\left(1-P_{w}\right)^{\bar{x}} \\
& N_{1}=n(0) \bar{x}\left(1-P_{w}\right)^{\bar{x}-1} P_{w} \\
& N_{2}=n(0) \frac{x(x-1)}{2}\left(1-P_{w}\right)^{\bar{x}-2} P_{w^{2}} \\
& \cdot \cdot \cdot \cdot \cdot \cdot \cdot \cdot \cdot \cdot \cdot \cdot \\
& N_{x}=n(0) P_{w}^{\bar{x}}
\end{aligned}
$$

As the value of $\boldsymbol{P}_{w}$ is generally known to be sufficiently less than unity, only $N_{0}$ and $N_{i}$ in eq 6 have contributed appreciably to $n(0)$. The theoretical equation corresponding to eq 6 would therefore be

$$
\begin{aligned}
\frac{f(t)}{f(0)}= & \left(1-P_{w}\right)^{\bar{x}} \exp \left(-k_{1} t\right) \\
& +\bar{x}\left(1-P_{w}\right)^{\bar{x}-1} P_{w} \exp \left(-k_{2} t\right)
\end{aligned}
$$

that is,

$$
\frac{f(t)}{f(0)}=A \exp (-k t)+B \exp \left(-k_{2} t\right)
$$

where,

$$
k_{1}=k \cdot \bar{x}, \quad k_{2}=k(\bar{x}-1)+K_{w}
$$

In eq $7^{\prime \prime}, k$ reprsents the specific rate constant for the major linkage and $k_{w}$ represents the specific rate constant for the weak linkage. As noted above, it was proved that the stress relaxation mechanism of cross-linked polymer, in which interchange reaction occurs, can be represented by the sum of two Maxwellian decay terms.

With reference to the sulfur-cured EPT (sample B), it was found that the cross-linking sites in these polymer networks consist of poly-, mono-, and di-sulfides as shown in Figure 9. In Figure $9, C(0)$ is the total of cross-linkages per cubic centimeter, $C_{1}(0)$ is the number of

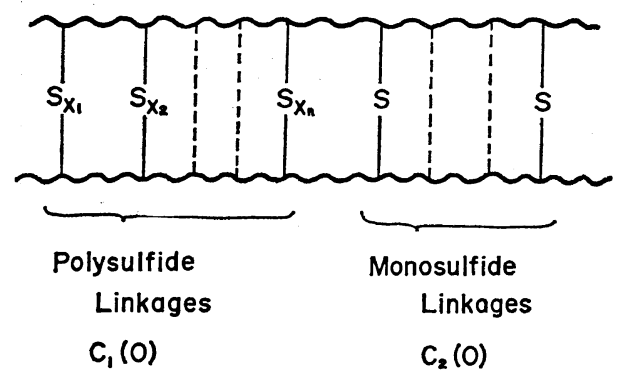

Figure 9. Structure of cross-linking sites. mono- and di-sulfide linkages. So when a chemorheological change occurs at the crosslinkages in sample $\mathbf{B}$, it is apparent for the above reason that $C_{1}(t)$ of sample $\mathrm{B}$ is connected with eq $7^{\prime}$ and $C_{2}(t)$ is related to eq 5 . Then the following equations are established.

$$
C(0)=C_{1}(0)+C_{2}(0)
$$

where,

$$
\begin{gathered}
\chi=C_{1}(0) / C(0) \\
C_{2}(0) / C(0)=1-\chi
\end{gathered}
$$

From eq 3,5 , and $7^{\prime}$

$$
\begin{aligned}
\frac{f(t)}{f(0)}= & A \exp \left(-k_{1} t\right)+B \exp \left(-k_{2} t\right) \\
& +(1-\chi) \exp (-k t)
\end{aligned}
$$

It has become apparent that for the chemical stress relaxation of the sulfur-cured EPT (sample B), the relation between relative stress $f(t) / f(0)$ and time $t$ is represented by eq 11 . That is, because eq 11 contains three Maxwellian decay terms, it can be divided into three straght lines by using Procedure $X$. So question II is solved.

Next, the answer to question I, that is why the straight lines on the long time side have a slightly descendent slope is based on eq 5 or on the third term in eq 11. This term corresponds to oxidative cleavages of mono- and di-sulfide linkages. So it is seen that as such a scission of cross-link sites has been neglected in Tobolsky's paper, ${ }^{3}$ the stress decay curve has become parallel to the abscissa. Finally the answer to question III is given as follows. According to Tobolsky, et al.,'s theory, ${ }^{1}$ in the case when the scission occurs only at cross-linkage in the cross-linked polymer, it is recognized that the decay curves of $f(t) / f(0) v s$. time are identical and independent of the cross-linking density $n(0)$. Tobolsky's theory supports our results in that the value of $k$ in eq 5 or on the third term in eq 5 is constant of $n(0)$ or $C_{2}(0)$, because the slopes of the straight lines described above all have the same decendent slopes independent of $n(0)$.

From the above experimental results it is clear that the scission of the cross-linkage occurs slightly in the TT-cured EPT (sample C). On the other hand, it has been elucidated that in natural rubber cured by irradiations, the scission of the main chain occurs only under the above 


\section{K. Murakami and S. Tamura}

conditions. ${ }^{7}$

In the case when both scissions of main chain and cross-linkage in the cross-linked polymer occur at the same time, the relationship between the change of chemical structure and the property of the polymer network chain have still not been make clear. Therefore, in order to investigate the degree of agreement between the theoretical relation for both scissions proposed by $\mathrm{us}^{8}$ and the experimental results, we carried out further studies. A TT-cured natural rubber (NR) (sample no. E) was prepared as a model polymer in which both scissions of main chain and cross-linkage occur at the same time. Initial density of this rubber was $\boldsymbol{n}^{\prime \prime}(0)$. At first, in the TT-cured EPT (sample no. C) which is a model polymer, only cross-link site scission occurs if the initial cross-link density of this polymer is $n(0)$, the number of cross-link scission $q_{c}(t)$ being represented by eq 2 described above. This equation is shown here again,

$$
q_{c}(t)=C(0)\left(1-\mathrm{e}^{-k t}\right)
$$

In the irradiation-cured natural rubber which is a model polymer, only main chain scission occurs (sample no. D) if the initial cross-link density is $n(0)$, the number of main chain scission $q(t)$ being represented by eq 12 as wellknown. ${ }^{10}$

$$
q(t)=-n^{\prime}(0) \ln \frac{f(t)}{f(0)}
$$

In the case of sample no. $E$ in which both scissions of main chain cross-linkage occur at the same time, if we consider the chemical stress relaxation of the above three samples under the same conditions, i.e., in the air at $190^{\circ} \mathrm{C}$, the following equation can be applied.

$$
\begin{aligned}
Q(t) & =q(t)+\frac{n^{\prime \prime}(0)}{n(0)} q_{c}(t) \\
& =-n^{\prime}(0) \ln \frac{f(t)}{f(0)}+\frac{n^{\prime \prime}(0)}{n(0)} C(0)\left(1-\mathrm{e}^{-k t}\right) \\
& =-n^{\prime}(0) \ln \frac{f(t)}{f(0)}+\frac{n^{\prime \prime}(0)}{2}\left(1-\mathrm{e}^{-k t}\right)
\end{aligned}
$$

Here, $Q(t)$ is the number of both scissions of main chains and cross-link sites for sample no. E. The value of $Q(t)$ of sample no. E under this condition can be determined from the calculation of eq 13 using the experimental results of both samples C and D. Furthermore, the next equation 14 was derived for the case in which both scissions of main chain and crosslinkage occur at the same $\operatorname{time}^{8}$ (refer to Appendix).

$$
\begin{aligned}
\frac{f(t)}{f(0)}= & -\frac{4 k(t) x}{k(t)+2 x} \frac{Q(t)}{M_{0}} \exp \left\{-\frac{2 x}{k(t)+2 x} \frac{Q(t)}{M_{0}}\right\} \\
& +\frac{2 k(t) x}{k(t)+2 x} \frac{Q(t)}{M_{0}} \exp \left\{-\frac{4 x^{2}}{k(t)+2 x} \frac{Q(t)}{M_{0}}\right\}
\end{aligned}
$$

where-

$$
\begin{aligned}
k(t) & =\frac{q_{c}(t) / C(0)}{q(t) M_{0}} \\
M_{0} & =x n(0)
\end{aligned}
$$

$k(t)$ is the ratio of the probability of cross-link scission to that of the main chain, $M_{0}$ is the total number of monomer units per cubic centimeter of cross-linked polymer and $x$ is the number of monomer units between the two cross-linkages. In eq 14 for sample $\mathrm{E}$, as the

Table II. Experimental values of $q_{c}(t), q(t)$, and $Q(t)$, and calculated values of $f(t) \mid f(0)$ obtained from eq 14

\begin{tabular}{lcccc}
\hline$t, \mathrm{hr}$ & $q_{c}(t) \times 10^{-7}$ & $q(t) \times 10^{-6}$ & $Q(t) \times 10^{-6}$ & $f(t) / f(0)$ \\
\hline 0 & 0 & 0 & 0 & 1.000 \\
0.5 & 0.05 & 4.7 & 4.7 & 0.963 \\
1 & 0.16 & 8.0 & 8.0 & 0.937 \\
1.5 & 0.31 & 9.8 & 9.8 & 0.925 \\
2 & 0.62 & 11.7 & 11.8 & 0.909 \\
3 & 1.24 & 14.8 & 14.9 & 0.888 \\
4 & 1.86 & 18.2 & 18.4 & 0.863 \\
5 & 3.09 & 21.6 & 21.9 & 0.839 \\
6 & 4.02 & 25.0 & 25.4 & 0.817 \\
\hline
\end{tabular}

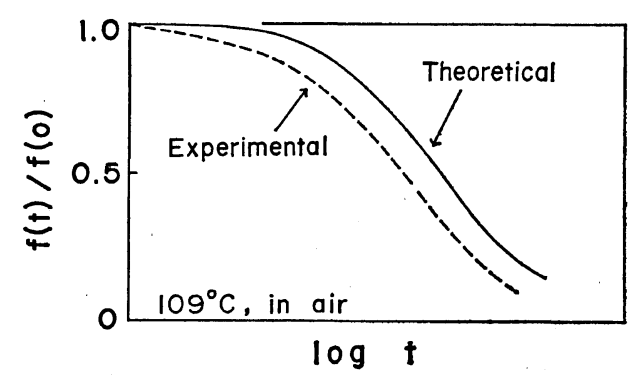

Figure 10. Calculated and observed stress relaxation curves of TT-cured natural rubber (sample no. E). 
experimental values of $Q(t), q_{c}(t), M_{0}$ and $k(t)$ are already known as shown above, and are tabulated in Table II, the relation between $f(t) / f(0)$ and $t$ of the sample $\mathrm{E}$ is predicted in Figure 10 as the calculated curve. From Figure 10, the observed curve lies usually lower than the calculated one and there is a significant difference between both curves. The main reason for this discrepancy is considered to be based upon the small change of the strength of the partial main chains near the cross-linkages, which has still not been made clear quantitatively. With more details, the changes of cross-linkage have the effect of making the partial main chains near the cross-linkages more subject to oxidative scission.

\section{APPENDIX8}

We considered the case of the tetrahedral model as the smallest unit of perfect network structure. When both scissions of the main chain and cross-linkage occur at the same time in the usual cross-linked polymer having a perfect network structure, the theoretical treatment is as follows. As defined in the text, $k$ is the ratio of the probability of cross-link scission to that of main chain scission. Consequently, $k=$ 0 ; when only main chain scission occurs and $k=\infty$; when only cross-linkage scission occurs. The equations defined in the text are shown once again.

$$
\begin{gathered}
Q(t)=q(t)+q_{c}(t) \\
k(t)=\frac{q_{c}(t) /\left(n^{\prime}(0) / 1\right)}{q(t) / M_{0}} \\
M_{0}=x n(0)
\end{gathered}
$$

It is now assumed that the cross-linkage scission occuring is unity or not more than unity at the smallest unit of network structure. (As the number of molecular scissions is natural number, the expression "scission is not more than unity", means that the number of molecular scissions is not more than that of the units of the smallest model in the plural network structure.) The above assumption is expressed by the eq 4 .

$$
\frac{q_{c}(t)}{n(0) / 2} \leqq \frac{1}{5}
$$

Substituting eq 1, 2, and 3 into eq 4, eq $4^{\prime}$ is obtained.

$$
\underline{q(t)} \bar{M}_{0} \leqq \frac{1}{5}\left(\frac{1}{k}+\frac{1}{2 x}\right)
$$

On the other hand, from eq 1,2 , and 3 ,

$$
\begin{aligned}
q(t) & =\frac{2 x}{2 x+k} Q(t) \\
q_{c}(t) & =\frac{k}{2 x+k} Q(t)
\end{aligned}
$$

Though both scissions of main chain and crosslinkage occur at the same time, we assume for convenience of calculation that the scissions occur firstly at the cross-linkage, i.e., $x$-mer and $2 x$-mer network chains appear owing to the changes after $t$ hours under the conditions of eq 4. The number of $x$-mer chains is $n(0)-$ $4 q_{c}(t)=n_{x}(0)$ and that of $2 x$-mer chains is $2 q_{c}(t)$. Next, considering the scission of the main chain, the number of $x$-mer chain scissions $q_{x}(t)$ is,

$$
q_{x}(t)=q(t) \frac{x n_{x}(0)}{M_{0}}
$$

That of $2 x$-mer chain scissions $q_{2 x}(t)$ is

$$
q_{2 x}=q(t) \frac{4 x q_{c}(t)}{M_{0}}
$$

Therefore, the number of residual $x$-mer chains after $t$ hours, $n_{x}(t)$ is obtained as follows.

$$
n_{x}(t)=n_{x}(0)\left\{1-\frac{q_{x}(t)}{n_{x}(0)}\right\}^{x} \approx n_{x}(0) \mathrm{e}^{-q_{x}(t) / n_{x}(0)}
$$

Similarly, that of residual $2 x$-mer chains $n_{2 x}(t)$ becomes,

$$
n_{2 x}(t)=2 q_{c}(t)\left\{1-\frac{q_{2 x}(t)}{4 x q_{c}(t)}\right\}^{2 x} \approx 2 q_{c}(t) \mathrm{e}^{-q_{2 x}(t) / 2 q_{c}(t)}
$$

The total number of effective network chains is

$$
n(t)=n_{x}(t)+n_{2 x}(t)
$$

Consequently, the relative stress, $f(t) / f(0)$ can be expressed by eq 12 .

$$
\begin{aligned}
\frac{f(t)}{f(0)} & =\frac{n(t)}{n(0)}=\frac{n_{x}(t)+n_{2 x}(t)}{n(0)} \\
& =\frac{1}{n(0)} n_{x}(0) \mathrm{e}^{-q_{x}(t) / n_{x}(0)}+2 q_{c}(t) \mathrm{e}^{-q_{2 x}(t) / 2 q_{c}(t)}
\end{aligned}
$$


Substituting eq 5 and 10 into eq 12 ,

$$
\begin{aligned}
\frac{n(t)}{n(0)}= & \frac{f(t)}{f(0)} \\
= & 1-\frac{4 k(t) x}{k(t)+2 x} \frac{Q(t)}{M_{0}} \exp \left\{-\frac{2 x}{k(t)+2 x} \frac{Q(t)}{M_{0}}\right\} \\
& +\frac{2 k(t) x}{k(t)+2 x} \frac{Q(t)}{M_{0}} \exp \left\{-\frac{4 x^{2}}{k(t)+2 x} \frac{Q(t)}{M_{0}}\right\}
\end{aligned}
$$

That is, eq 13 in the Appendix corresponds to eq 14 in the text. Substituting $k=0$ into eq 13, in other words, in the case of main chain scission only, eq $13^{\prime}$ is obtained.

$$
\frac{n(t)}{n(0)}=\frac{f(t)}{f(0)}=\mathrm{e}^{-q(t) / n(0)}
$$

On the other hand, substituting $k=\infty$ in eq 13, in other words, in the case of the cross-linkage scission only, eq $13^{\prime \prime}$ is obtained.

$$
n(0)=n(t)+2 Q(t)
$$

Both eq $13^{\prime}$ and $13^{\prime \prime}$ are entirely in agreement with the equations proposed by Tobolsky.

Furthermore, in such a similar model, when assuming the case that the cross-linkage scissions are more than unity and less than two under the conditions of the following eq 14 , eq 15 is derived which is somewhat more complicated compared with eq 13 .

$$
\begin{gathered}
\frac{1}{5}<\frac{q_{c}(t)}{n(0) / 2} \leqq \frac{2}{5} \\
\frac{n(t)}{n(0)}=\frac{f(t)}{f(0)}=\left\{1-\frac{7}{2} \frac{k(t) x}{k(t)+2 x} \frac{Q(t)}{M_{0}}\right\} \\
\times \exp \left\{-\frac{2 x^{2}}{k(t)+2 x} \frac{Q(t)}{M_{0}}\right\}
\end{gathered}
$$

$$
\begin{aligned}
& +\frac{k(t) x}{k(t)+2 x} \frac{Q(t)}{M_{0}} \exp \left\{-\frac{4 x^{2}}{k(t)+2 x} \frac{Q(t)}{M_{0}}\right\} \\
& +\frac{k(t) x}{2 k(t)+4 x} \frac{Q(t)}{M_{0}} \exp \left\{-\frac{6 x^{2}}{k(t)+2 x} \frac{Q(t)}{M_{0}}\right\}
\end{aligned}
$$

Here, from the Table II in the text, in our experiment for TT-cured natural rubber (sample E), $q_{c}(t) /(n(0) / 2$ is obviously very small and adequately satisfies the condition in eq 4 . Consequently, it is clear that eq 13 in the Appendix as used in the text is reasonable and satisfactory.

\section{REFERENCES}

1. A. V. Tobolsky, J. Appl. Phys., 27, 673 (1956); A. V. Tobolsky, "Properties and Structure of Polymers, John Wiley, New York, N.Y., 1960, p 228.

2. A. V. Tobolsky, A. Mercurio, J. Polym. Sci., 36, 467 (1959).

3. P. F. Lyons, T. C. P. Lee, and A. V. Tobolsky, Rubber Chem. Tech., 39, 1634 (1966).

4. A. V. Tobolsky and K. Murakami, J. Polym. Sci., 40, 443 (1959); A. V. Tobolsky, "Properties and Structure of Polymers," John Wiley, New York, N. Y., 1960, p 188.

5. A. V. Tobolsky, Polym. Letters, 2, 823 (1964).

6. R. B. Beevers, J. Colloid Sci., 19, 40 (1964).

7. A. V. Tobolsky, Properties and Structure of Polymers, John Wiley, New York, N.Y., 1960, p 209.

8. K. Murakami, S. Tamura, Y. Takano, and H. Kurumiya, Kobunshi Kagaku (Chem. High Polymers), 24, 699 (1967); K. Murakami and S. Tamura, Kobunshi Kagaku (Chem. High Polymers), 24, 706 (1967).

9. M. D. Stern and A. V. Tobolsky, J. Chem. Phys., 14, 93 (1946).

10. A. V. Tobolsky, J. Appl. Phys., 27, 673 (1956). 